\title{
Synovial bone sialoprotein indicates aseptic failure in total joint arthroplasty
}

\author{
André Busch ${ }^{1,2}$, Marcus Jäger ${ }^{1,2}$, Florian Dittrich ${ }^{3}$, Alexander Wegner ${ }^{1,2}$, Stefan Landgraeber ${ }^{3}$ and
}

Marcel Haversath ${ }^{4 *}$

\begin{abstract}
Background: Until today, a reliable diagnostic discrimination between periprosthetic joint infections (PJI) and aseptic failure (AF) after total joint arthroplasty (TJA) remains challenging. Nearly all recent research focused on synovial markers to be elevated in PJI rather than in AF patients. In this study, synovial bone sialoprotein (sBSP) was investigated in PJI and AF arthroplasty patients before revision surgery.

Methods: SBSP and C-reactive protein (CRP) were determined in synovial fluid samples of PJI $(n=13)$ patients fulfilling the MSIS criteria and AF ( $n=25)$ patients. Beside descriptive analysis and comparison, computed statistics determined the area under the receiver operating characteristics curve (AUC) to evaluate the discrimination ability of the tested synovial markers.

Results: In patients with PJI according to the MSIS criteria, mean sBSP was significantly lower: $14.8 \mathrm{ng} / \mathrm{ml}(95 \% \mathrm{Cl}$ 5.5-24.1) vs. $38.2 \mathrm{ng} / \mathrm{ml}$ in the AF group ( $95 \% \mathrm{Cl} 31.1-45.3), p \leq 0.001$. Conversely, mean sCRP was significantly higher in PJl patients: $8.4 \mu \mathrm{g} / \mathrm{ml}(95 \% \mathrm{Cl} 0-17.2)$ vs. $1.8 \mu \mathrm{g} / \mathrm{ml}$ in the AF group ( $95 \% \mathrm{Cl} 0.9-2.8), p=0.032$. The AUC of sCRP in PJI patients was 0.71 . The AUC of SBSP in AF revision arthroplasty patients was 0.83 . The detection of osteolyses was not associated with higher sBSP concentrations.

Conclusions: Considering the MSIS criteria, significantly higher SBSP concentrations were found in synovial fluid samples of AF compared to PJI patients. SCRP showed only fair, SBSP good discrimination potential. If it is not clear whether PJI is present or not, SBSP may be considered as an add-on synovial marker.
\end{abstract}

\section{Introduction}

Periprosthetic joint infection (PJI) is a severe complication after total joint arthroplasty. It is the third leading cause for revision surgery in failure hip arthroplasty [1]. The 5-year incidence rate exceeds one percent following the primary procedure. Not only in the USA but worldwide, revision arthroplasty is predicted to grow considerably in the next decades. Among others, notable risk factors for the development of periprosthetic joint infections are internal comorbidities, male gender, overweight, and prolonged surgery time. The differentiation

\footnotetext{
* Correspondence: marcel.haversath@vkkd-kliniken.de

${ }^{4}$ Department of Orthopaedics, St. Vinzenz-Krankenhaus, Schloßstraße 85, 40477 Düsseldorf, Germany

Full list of author information is available at the end of the article
}

between aseptic and septic failure is crucial for surgical planning. According to the International Consensus Group, a minimum of two positive cultures of periprosthetic tissue or the presence of a sinus tract with evidence of communication to the joint or visualization of the prothesis are major criteria in the diagnosis of PJI [2]. A major problem remains that microbiological cultures still produce false negative or positive results. Besides white cell count and C-reactive protein (CRP), other, more sensitive and specific serum or synovial biomarkers are in focus of current research [3-5]. For instance, Procalcitonin and Interleukin-6, which are commonly used to evaluate inflammation processes, were investigated toward their reasonable determination in PJI diagnostics. However, they also reveal deficits in 
sensitivity and specificity [3]. Alpha defensin is another synovial marker that has found its way to the market with a quantitative laboratory ELISA and a qualitative quick test that is designated as an aid in the intraoperative diagnosis of PJI (Synovasure ${ }^{\bullet}$ alpha-defensin test, Zimmer Biomet). The latest meta-analysis revealed a promising diagnostic sensitivity and specificity of alpha defensin in PJI diagnostics [6]. Conversely, other authors attested a poor test sensitivity independent of the test method, quantitative or qualitative $[7,8]$. Yet, there is no "gold standard" in serum or synovial fluid biomarkers for reliable diagnosis of PJI [9]. On the other side, there are no reliable synovial markers that indicate aseptic TJA failure.

Bone sialoprotein (BSP) is a glycoprotein that is only found in the extracellular matrix of bone and dentine [10]. High concentrations of BSP are located in the osteoid, the newly formed bone tissue of growing bone, which is the most common site for osteomyelitis [11]. It has been shown that BSP selectively binds to staphylococci isolated from patients suffering from osteomyelitis and septic arthritis [12]. The bacterial cell wall glycoprotein BSP-binding protein (Bbp) induces an immune response and elevated serum IgG-antibodies to Bbp were found to be related to $S$. aureus osteomyelitis of the diabetic foot [13]. Due to the bacterial binding of BSP, we speculated to detect lower synovial levels in PJI patients.

The purpose of this study was to investigate the diagnostic and prognostic value of BSP in synovial fluid for the diagnosis of aseptic failure TJA. Furthermore, synovial BSP (sBSP) was compared to the already evaluated synovial C-reactive protein (sCRP) and other than sBSP known to be enhanced in PJI patients.

\section{Material and methods Study design}

This research has been approved by the IRB of the authors' affiliated institutions.

Preoperatively, the medical history was recorded and clinical examination, laboratory values including serum CRP and joint aspiration fluid were investigated as routine diagnostic procedures in revision arthroplasty of the hip, knee, and shoulder. Furthermore, preoperative Xrays were analyzed toward manifest osteolyses by three independent observers (all orthopedic surgeons). Inclusion criteria were an adequate synovial fluid volume for laboratory marker measurements as well as full clinical and laboratory data to allow the diagnosis of PJI. Patients suffering from systemic inflammatory diseases (SID) were also included. Patients receiving antibiotics before joint aspiration and cases of early postoperative PJI ( 8 weeks) were excluded because of the lack of reliability in the determination of synovial and serologic markers shortly after surgery $[14,15]$.
The differential diagnosis between aseptic and septic was made according to the musculoskeletal infection society (MSIS) excluding the sedimentation rate as a minor criterion $[16,17]$.

\section{Patients}

From July 2018 to March 2019, 38 patients received revision arthroplasty and were included in the study. Written informed consent to participate in the study was obtained prior to intervention. Tables 1 and 2 summarize the main patient characteristics.

Of these 38 patients, 13 met the abovementioned criteria for PJI. There were six hips, six knees, and one shoulder. The PJI group included eight women and five men with a mean age of 76 years \pm 8.5 (55-88). In this group, the synovial fluid of eleven patients was tested positive in the microbiological culture. Organisms include Staphylcocci $(5 \times)$, Klebsiella species (3x), Pseudomonas (1x), Cutibacterium acnes $(1 \times)$ and Escherichia coli $(1 \times)$. One patient $(8 \%)$ presented with a sinus tract. None of the patients were treated with antibiotics prior to culture sampling. Radiological signs of prosthetic loosening could be found in ten patients.

On the other side, 19 women and six men were included in the aseptic control group. There were 14 hips, nine knees, and two shoulders. In the control group, 15 patients suffered from polyethylene wear debris induced osteolysis. Fifteen patients $(60 \%)$ received exchange of the polyethylene liner and head. Radiological signs of prosthetic loosening could be found and in ten patients. In two hips, corrosion of the modular head-neck junction could be found. Four patients suffered from spinal column associated pain and the diagnostic puncture of the hip ( $3 \times$ inset THA) or knee ( $1 \times$ inset TKA) was performed for differential diagnosis. Five patients of the control and one of the PJI group reported a systemic inflammatory disease before revision arthroplasty.

\section{Sample preparation and biomarker analysis}

Synovial aspiration was performed under sterile conditions avoiding an admixture of blood with an 18-gage needle prior to surgical intervention.

Synovial fluid was aliquoted into sterile tubes and immediately put on ice. The synovial fluid samples were transported to the laboratory within $60 \mathrm{~min}$ and frozen and stored at $-80^{\circ} \mathrm{C}$.

The immunoassay for synovial fluid BSP and synovial CRP was performed according to manufacturer's specification using reagents from LifeSpan Bio (Seattle, USA). The specimens were measured in duplicates by standard enzyme-linked sandwich immunosorbent assay. 
Table 1 Main patient characteristics

\begin{tabular}{|c|c|c|c|c|c|c|}
\hline & \multicolumn{3}{|c|}{ PJl patients (according to MSIS) } & \multicolumn{3}{|c|}{ Aseptic failure of arthroplasty } \\
\hline Number $(n)$ & \multicolumn{3}{|l|}{13} & \multicolumn{3}{|l|}{25} \\
\hline Gender ratio $(m / f)$ & \multicolumn{3}{|l|}{$5 / 8$} & \multicolumn{3}{|l|}{$6 / 19$} \\
\hline MSIS major criteria & \multicolumn{3}{|l|}{$12 / 13$} & \multicolumn{3}{|l|}{-} \\
\hline \multicolumn{7}{|l|}{ Affected joint } \\
\hline Hip/knee/shoulder & \multicolumn{3}{|l|}{$6 / 6 / 1$} & \multicolumn{3}{|c|}{$14 / 9 / 2$} \\
\hline \multicolumn{7}{|l|}{ Radiological evidence of prosthetic loosening } \\
\hline - Cup (hip) & \multicolumn{3}{|l|}{1} & \multicolumn{3}{|l|}{4} \\
\hline - Stem (hip) & \multicolumn{3}{|l|}{4} & \multicolumn{3}{|l|}{3} \\
\hline - Femoral component (knee) & \multicolumn{3}{|l|}{3} & \multicolumn{3}{|l|}{1} \\
\hline - Tibial component (knee) & \multicolumn{3}{|l|}{4} & \multicolumn{3}{|l|}{0} \\
\hline - Glenoid/glenosphere (shoulder) & \multicolumn{3}{|l|}{1} & \multicolumn{3}{|l|}{0} \\
\hline - Humeral component (shoulder) & \multicolumn{3}{|l|}{1} & \multicolumn{3}{|l|}{3} \\
\hline \multirow[t]{2}{*}{ Osteolyses } & Mild & Moderate & Severe & Mild & Moderate & Severe \\
\hline & 4 & 3 & 3 & 8 & 7 & 2 \\
\hline \multicolumn{7}{|l|}{ Bacteria detection in synovial fluid } \\
\hline - S. aureus & \multicolumn{3}{|l|}{2} & \multicolumn{3}{|l|}{-} \\
\hline - S. epidemidis & \multicolumn{6}{|l|}{2} \\
\hline - S. warneri & \multicolumn{6}{|l|}{1} \\
\hline - Klebsiella pneumoniae & \multicolumn{6}{|l|}{3} \\
\hline - Pseudomonas aeruginosa & \multicolumn{6}{|l|}{1} \\
\hline - Cutibacterium acnes & \multicolumn{6}{|l|}{1} \\
\hline - E. coli & 1 & & & & & \\
\hline - None & 2 & & & & & \\
\hline Evidence of systemic inflammatory disease & 1 & & & 5 & & \\
\hline
\end{tabular}

\section{Statistical analysis}

The data was analyzed using SPSS Statistics 26 (IBM, Armonk, NY, USA). Descriptive statistics was used to analyze clinical and laboratory values. Statistical analysis indicated a non-parametric distribution of sBSP and sCRP in PJI and AF patients. Therefore, the MannWhitney $U$ test was used to compare independent samples. For sensitivity, specificity and area under the curve (AUC) calculation, a receiver operating characteristics curve analysis was performed. $P$ values below 0.05 were considered as statistically significant, values below 0.01 as highly significant.

\section{Results}

\section{SBSP and SCRP behave in opposite ways}

The synovial bone sialoprotein and CRP results are presented in Fig. 1 (Fig. 1a, b). In patients with PJI according to the MSIS criteria, mean sBSP was significantly lower than in the aseptic group: $14.8 \mathrm{ng} / \mathrm{ml}$ (95\% CI 5.5$24.1)$ vs. $38.2 \mathrm{ng} / \mathrm{ml}$ (95\% CI 31.1-45.3), $p \leq 0.001$. Conversely, mean sCRP was significantly higher in PJI patients: $8.4 \mu \mathrm{g} / \mathrm{ml}(95 \% \mathrm{CI} 0-17.2)$ vs. $1.8 \mu \mathrm{g} / \mathrm{ml}$ in the control group (95\% CI 0.9-2.8), $p=0.032$.

In patients suffering from SID, mean sCRP was $3.3 \mu \mathrm{g} /$ $\mathrm{ml}$ and in those who did not report SID it was $4.2 \mu \mathrm{g} / \mathrm{ml}$ $(p=0.80)$.

\section{Discrimination ability of sBSP and sCRP}

The AUC of sBSP in aseptic revision arthroplasty patients was 0.83 (Fig. 2a). The AUC of sCRP in PJI patients was 0.71 (Fig. 2b). sBSP values of $24.4 \mathrm{ng} / \mathrm{ml}$ and above had a sensitivity of 0.69 and specificity of 0.80 in the detection of AF patients. The ROC curve analysis of sCRP revealed at a high specificity level of 0.92 , a very poor test sensitivity of 0.31 (CRP threshold: $5.08 \mu \mathrm{g} / \mathrm{ml}$ ).

SBSP in joint nearby osteolyses, in Staphylococci induced $\mathrm{PJI}$ and systemic inflammatory disease

As classic bone matrix protein, high sBSP values were speculated in synovial fluid of patients with nearby osteolyses due to an increased release from the bone matrix. In the PJI group, ten patients (77\%) showed osteolytic lesions in conventional X-rays of the affected 
Table 2 Individual listing of patients. Reasons for revision arthroplasty and surgical interventions after joint puncture. PJI patients are highlighted in gray

\begin{tabular}{|c|c|c|c|c|}
\hline Patient & $\begin{array}{l}\text { Age (at time } \\
\text { of surgery) }\end{array}$ & Gender & Reason for surgery & Intervention carried out (after joint puncture) \\
\hline$\# 1$ & 63 & $\mathrm{~F}$ & PJI & THA explantation + antibiotic spacer \\
\hline \#2 & 88 & $\mathrm{~F}$ & PJI & THA explantation + antibiotic spacer \\
\hline \#3 & 79 & M & PJI & TSA explantation + antibiotic spacer \\
\hline \#4 & 84 & $\mathrm{~F}$ & PJI & THA explantation + antibiotic spacer \\
\hline \#5 & 77 & M & PJI & TKA explantation + antibiotic spacer \\
\hline \#6 & 76 & $\mathrm{~F}$ & PJ & THA explantation + antibiotic spacer \\
\hline \#7 & 76 & $\mathrm{~F}$ & PJI & THA explantation + antibiotic spacer \\
\hline \#8 & 63 & $\mathrm{~F}$ & PJI & TKA explantation + antibiotic spacer \\
\hline \#9 & 77 & M & PJI & TKA explantation + antibiotic spacer \\
\hline \#10 & 81 & M & PJI & TKA explantation + antibiotic spacer \\
\hline \#11 & 76 & $\mathrm{~F}$ & PJI & THA explantation + antibiotic spacer \\
\hline$\# 12$ & 75 & $\mathrm{~F}$ & PJI & $\begin{array}{l}\text { exchange of antibiotic spacer after THA explantation } \\
+ \text { antibiotic spacer }\end{array}$ \\
\hline$\# 13$ & 79 & $\mathrm{~F}$ & PJI & THA explantation + antibiotic spacer \\
\hline$\# 14$ & 55 & $\mathrm{~F}$ & $\begin{array}{l}\text { Intraarticular free-floating cement-related } \\
\text { particles after TKA }\end{array}$ & Removal of cement-related particles + inlay exchange \\
\hline \#15 & 79 & M & Knee instability + scarred adhesions & Revision total knee arthroplasty \\
\hline \#16 & 45 & $\mathrm{~F}$ & Aseptic cup loosening & Revision hip arthroplasty of cup and femoral head \\
\hline$\# 17$ & 79 & $\mathrm{~F}$ & Liner wear & Revision hip arthroplasty of femoral head and liner \\
\hline \#18 & 79 & $\mathrm{~F}$ & Recurrent hip dislocation & Revision hip arthroplasty of cup and femoral head \\
\hline \#19 & 51 & M & Aseptic femoral and tibial loosening & Revision total knee arthroplasty \\
\hline \#20 & 81 & $\mathrm{~F}$ & Periprosthetic fracture & Revision total shoulder arthroplasty \\
\hline \#21 & 63 & $\mathrm{~F}$ & Spinal column associated pain, bursitis trochanterica & No revision, diagnostic joint aspiration of the hip (inset THA) \\
\hline \#22 & 80 & $\mathrm{~F}$ & Spinal column associated pain & No revision, diagnostic joint aspiration of the knee (inset TKA) \\
\hline \#23 & 69 & $\mathrm{~F}$ & Spinal column associated pain & No revision, diagnostic joint aspiration of the hip (inset THA) \\
\hline \#24 & 79 & $\mathrm{~F}$ & Recurrent patellar instability + arthrofibrosis & Arthrolysis + revision total knee arthroplasty \\
\hline \#25 & 72 & M & Inlay wear, knee instability & Arthrolysis + inlay exchange \\
\hline \#26 & 43 & $\mathrm{~F}$ & $\begin{array}{l}\text { Particle disease, aseptic peritrochanteric } \\
\text { osteolysis }\end{array}$ & $\begin{array}{l}\text { Exchange of femoral head and liner and bone void filling } \\
\text { with synthetic CaP-cement }\end{array}$ \\
\hline \#27 & 83 & M & Aseptic femoral and tibial loosening & Total revision knee arthroplasty \\
\hline \#28 & 82 & $\mathrm{~F}$ & Knee instability & Revision of femoral component + inlay exchange \\
\hline \#29 & 83 & $\mathrm{~F}$ & Periprosthetic fracture & Revision total shoulder arthroplasty \\
\hline \#30 & 81 & $\mathrm{~F}$ & Periprosthetic fracture & Revision total shoulder arthroplasty \\
\hline \#31 & 68 & M & Lumboischialgia and hip pain & No revision, diagnostic joint aspiration (inset THA) \\
\hline \#32 & 78 & $\mathrm{~F}$ & Recurrent hip dislocations & Exchange of femoral head and inlay \\
\hline \#33 & 83 & M & Aseptic femoral and tibial loosening & Total revision knee arthroplasty \\
\hline \#34 & 82 & $\mathrm{~F}$ & Knee instability & Revision of femoral component + inlay exchange \\
\hline \#35 & 38 & $\mathrm{~F}$ & $\begin{array}{l}\text { Particle disease, aseptic osteolysis of the } \\
\text { greater trochanter }\end{array}$ & $\begin{array}{l}\text { Exchange of femoral head and inlay and bone void filling } \\
\text { with CaP bone cement }\end{array}$ \\
\hline \#36 & 88 & M & Aseptic femoral loosening & Revision of femoral stem, femoral head and liner \\
\hline \#37 & 52 & $\mathrm{~F}$ & Knee instability, inlay wear & Inlay exchange + partial patellar resection \\
\hline \#38 & 79 & $\mathrm{~F}$ & Aseptic acetabular loosening & Revision hip arthroplasty of cup \\
\hline
\end{tabular}



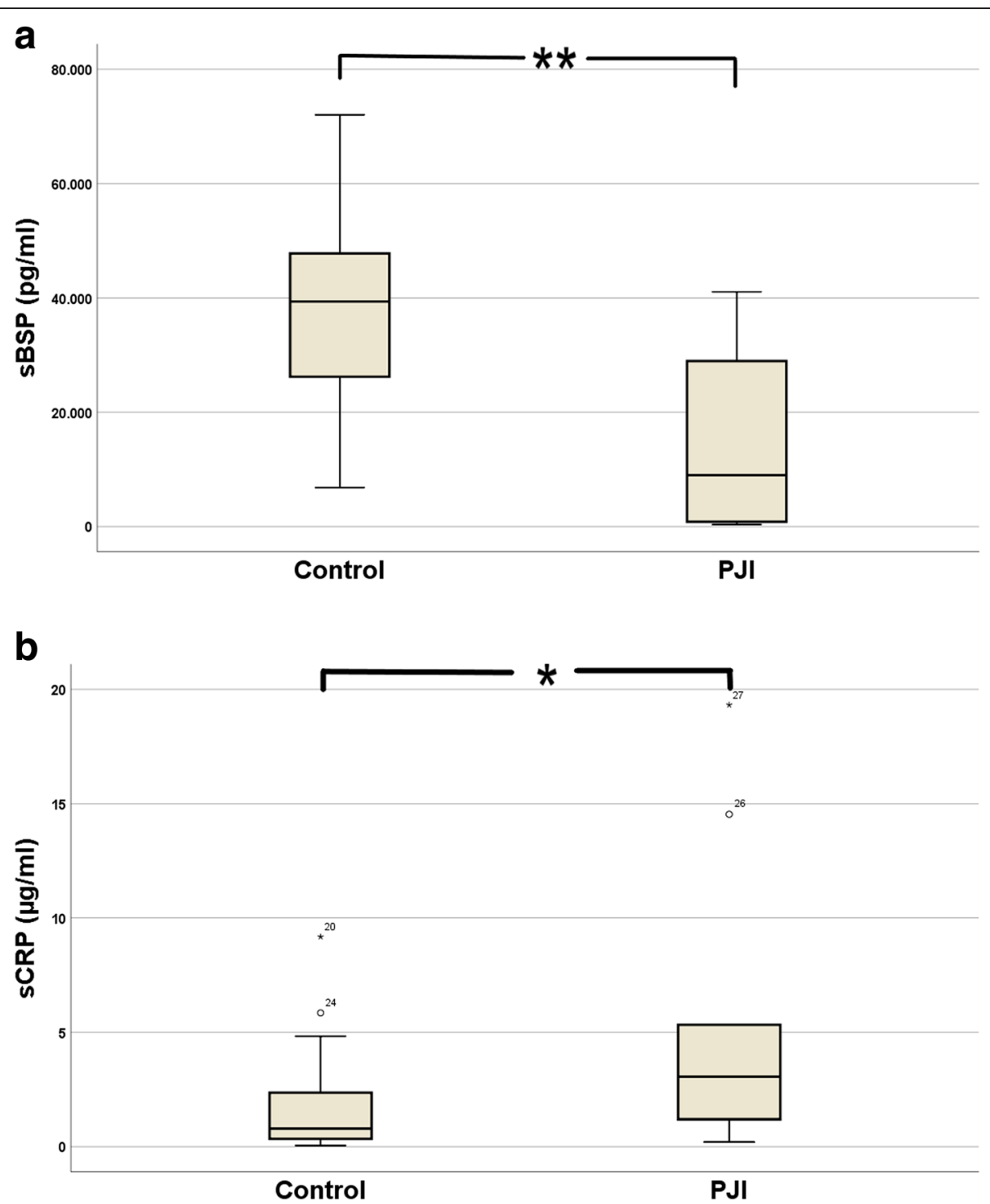

Fig. 1 Boxplot diagram. Demonstrates the concentrations of sBSP in the control and PJl group (a) and of sCRP (b), respectively. The difference between the groups was highly significant in $\operatorname{SBSP}\left({ }^{* *} p \leq 0.01\right)$ and significant in $\operatorname{SCRP}\left({ }^{*} p \leq 0.01\right)$

joint in two planes. In the control group, the rate of osteolytic lesions was $0.68(n=17)$. There was no significant difference of measured sBSP values between the detection of osteolyses (mean sBSP: $26.5 \mathrm{ng} / \mathrm{ml}$ ) or not (vs. $39.3 \mathrm{ng} / \mathrm{ml} ; p=0.071$ ). Fig. 3 demonstrates one case of a Pseudomonas aeruginosa PJI with severe osteolyses.

A tendency of higher mean sBSP values could be observed in Staphylococci-induced PJI compared to the group of other detected bacterial species $(26.0 \mathrm{ng} / \mathrm{ml}$ vs. $4.2 \mathrm{ng} / \mathrm{ml})$. This was a small sample comparison $(n=5$ vs. $n=6)$ and the difference was not significant ( $p=$ 0.126 ).

No difference of sBSP values in SID compared to nonaffected patients could be found $(30.1 \mathrm{ng} / \mathrm{ml}$ vs. $30.2 \mathrm{ng} /$ $\mathrm{ml} ; p=0.922)$.

\section{Discussion}

Until today, the diagnosis of periprosthetic joint infections remains challenging. The latest proposed algorithm for diagnosing PJI by Parvizi et al. still includes, beside physical examination, the long-established serum Creactive protein as a first step [18]. Recent research focused on novel serum or synovial biomarkers. Still, the aim of the search for suitable synovial biomarkers is to simplify PJI diagnostics.

Latest proteomic research of the synovial fluid identified lactoferrin, polymorphonuclear leukocyte serine protease 3, and myeloid nuclear differentiation antigen as potential candidates for reliable PJI diagnostics [19]. Others focused on synovial C-reactive protein, presepsin, or lipocalin-2 [5, 20, 21]. 

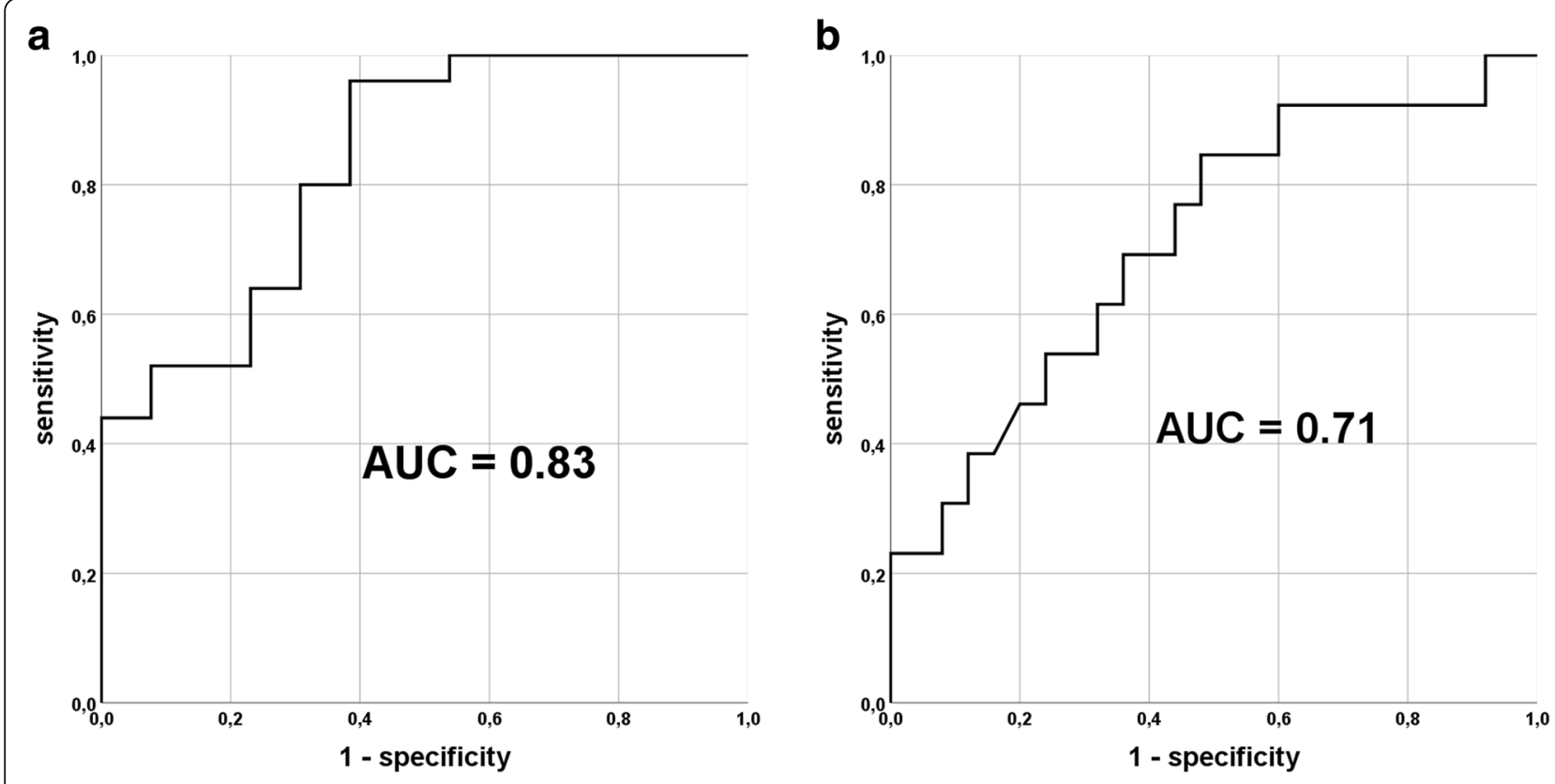

Fig. 2 ROC analysis and AUC of synovial BSP (a) high sBSP in aseptic patients and CRP (b) high sCRP in PJI patients

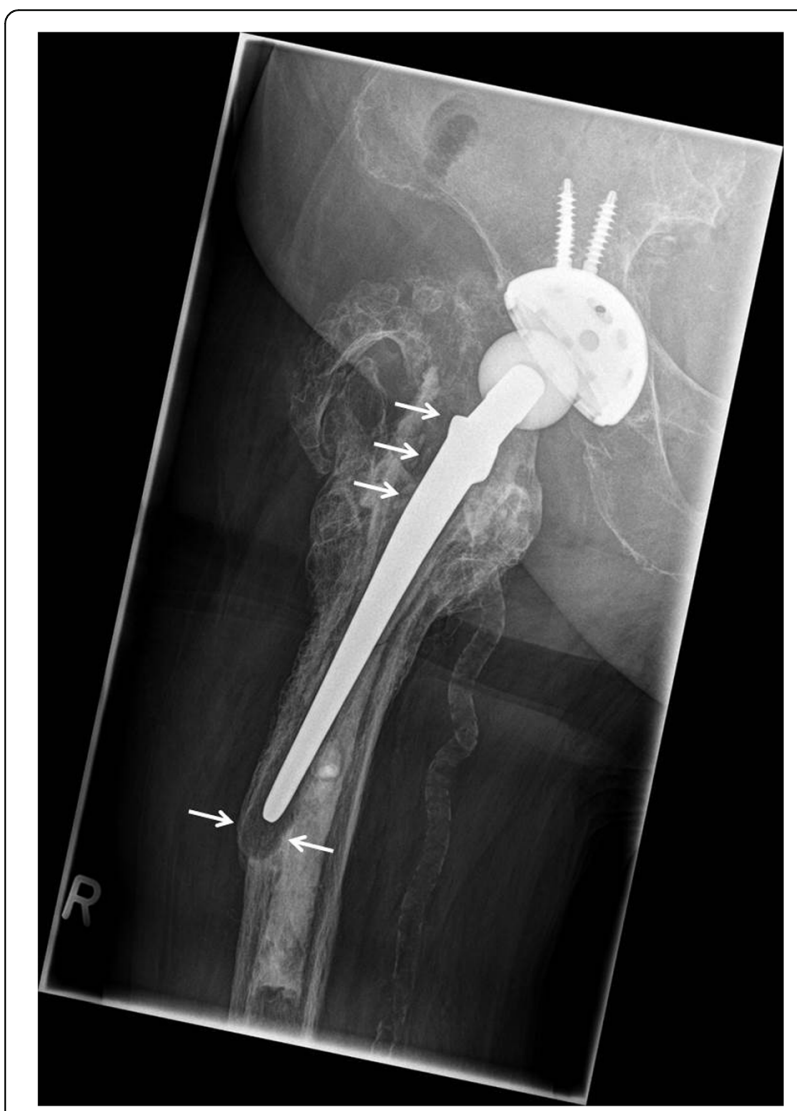

Fig. $3 \mathrm{PJl}$ of the right hip. Severe osteolyses of the cemented stem are visible (white arrows). Pseudomonas aeruginosa was detected in the synovial fluid. sBSP was measured low at $0.8 \mathrm{ng} / \mathrm{ml}$ and sCRP high at $14.5 \mu \mathrm{g} / \mathrm{ml}$
Against this backdrop, alpha-defensin was a promising candidate and is available on the market as a quick test. However, it is still not recommended in routine diagnostics, which might be a problem of its partially described poor sensitivity $[7,8,18]$.

Test accuracy of all those investigated novel synovial biomarkers is calculated by using different established classification systems. These were proposed, inter alia, by various societies such as the musculoskeletal infection society (MSIS), the infectious diseases society of America or the European bone and joint infection society [7].

The newly proposed "2018 Definition of Periprosthetic Hip and Knee Infection" claims a nearly equal specificity but a noticeably higher sensitivity than the International Consensus Meeting definition or the MSIS, which was used in this study [2]. However, due to an effortful score calculation, it remains to be seen whether such a score will prevail in clinical routine. And especially in cases of a failed articular puncture, an intraoperative quick test of a synovial fluid biomarker may help to gain safety for the surgeon and the patient.

Remarkably, recent research did not focus on synovial markers to be elevated in aseptic failure TJA.

BSP is a significant component of the bone extracellular matrix and has been suggested to constitute approximately $8 \%$ of all non-collagenous proteins found in bone [22]. Binding of Staphylococci to BSP in osteomyelitis was firstly described by Rydén et al. in 1987 [12]. Not only Staphylococcus aureus but also epidermidis was identified to show this behavior [10, 23]. Further research revealed that an adhesion protein, named bone 
sialoprotein-binding protein, expressed by Staphylococci and able to bind the most abundant bone proteins may play a crucial role in orthopedic implant infections [24]. Due to bacterial binding of BSP in PJI patients, we speculated elevated synovial BSP in cases of AF patients and the results met our expectations. In the PJI cohort, on average lower BSP values were measured in synovial fluid samples. However, we could not find a connection of sBSP to concomitant local osteolyses detected in conventional radiographs. Nevertheless, this study does not answer the question if the reason for lower average sBSP values in PJI patients is bacterial binding of sBSP. This needs to be investigated in further studies.

Worldwide, serum CRP is a well-known diagnostic and process parameter in inflammation and commonly used in the diagnostic algorithm of PJI [2].

Synovial CRP was also measured in the samples of this study. According to the MSIS criteria, sCRP was fairly capable in distinguishing between the classes with an AUC of 0.71 . In a recently published meta-analysis, synovial CRP was designated as a good biomarker for the diagnosis of PJI with high sensitivity and specificity [25]. The authors measured a pooled sensitivity of 0.92 , a specificity of 0.90 and an AUC of 0.966. Our results do not support the described high accuracy of sCRP in the diagnostic of PJI. At a sensitivity level of 0.92 and above the sensitivity was only 0.31 which would have resulted in high amounts of false-positive test results at a threshold of $5.08 \mu \mathrm{g} / \mathrm{ml} \mathrm{sCRP}$ in our cohort.

Other than sCRP or sBSP, alpha-defensin (sAD) is a microbicidal protein that is produced by neutrophils as a response to microbial products or proinflammatory cytokines. The promising results in its discrimination ability in PJI patients led to its increasing use in clinical practice. However, recent data support the fact of a high specificity but only poor sensitivity [8]. Our results of sCRP were not able to reach the good values of alphadefensin. Even at a sensitivity level of in part just over $50 \%$, sCRP was not able to reach a specificity of over 95\% likewise alpha-defensin (Fig. 2b).

Interestingly, from an economic point of view, a recently published meta-analysis suggests that a simple leukocytes esterase strip may have the same power compared to a cost-intensive SAD measurement [26].

Five patients of our control group $(20 \%)$ and one of the PJI patients $(7.7 \%)$ suffered from systemic inflammatory diseases. Compared to the non-affected patients, no significant differences of sBSP or sCRP measurements could be found. This is in accordance to recent research that demonstrated that concomitant SID may not affect the accuracy of infection biomarkers in patients with PJI [27].

Future research should not only focus on novel synovial markers to detect PJI or AF patients but also on serum markers. This would solve the problem of failed joint punctures and it would significantly increase patient comfort. Because almost 100 years after the introduction of erythrocyte sedimentation rate and 90 years after the discovery of C-reactive protein, both are still state of the art in serum PJI diagnostics [28-30].

Limitations of this study are the exclusion of the sedimentation rate in the PJI diagnosis of our cohort. This may have had an impact on the sensitivity of the MSIS proceedings and at worst causing a higher proportion of false-negative results. Moreover, the MSIS criteria used in this study were published in 2011 and recently a validated updated version was published claiming a higher sensitivity than before [2]. Last but not least, the small sample size and low homogeneity of the included patients may have had a negative influence on the statistical power of our results. We advocate further studies with a more homogeneous patient population and especially the study of patients with suspected low-grade infections that do not clearly meet the MISIS criteria. It is precisely with these patients that synovial BSP could provide a benefit in everyday clinical practice.

\section{Conclusions}

Considering the MSIS criteria, significantly higher sBSP concentrations were found in synovial fluid samples of AF compared to PJI patients. sCRP showed only fair, sBSP good discrimination potential. If it is not clear whether PJI is present or not, sBSP may be considered as an add-on synovial marker.

\section{Abbreviations \\ PJI: Periprosthetic joint infection; AF: Aseptic failure; TJl: Total joint arthroplasty; TSA: Total shoulder arthroplasty; THA: Total hip arthroplasty; TKA: Total knee arthroplasty; sBSP: Synovial bone sialoprotein; SCRP: Synovial C-reactive protein; MSIS: Muskuloskeletal infection society; AUC: Area under the curve; Bbp: Bone sialoprotein-binding protein; IRB: Institutional Review Board; SID: Systemic inflammatory disease; SAD: Synovial alpha-defensin; CaP: Calcium phosphate}

\section{Acknowledgements \\ Not applicable}

\section{Authors' contributions}

$A B$ initiated the study, created ethics proposal, and enrolled patients. $\mathrm{MH}$ wrote the main manuscript and created table and figures. MJ analyzed the data and supported $\mathrm{MH}$ in writing the paper. FD enrolled patients and performed joint aspiration. SL and AW supported lab work and statistical analysis. All authors read and approved the final manuscript.

\section{Funding}

The authors received no specific funding for this work.

\section{Availability of data and materials}

The datasets analyzed during the current study are available from the corresponding author on reasonable request.

Ethics approval and consent to participate

Prior starting this prospective study, approval from the local ethics committee (no. 18-8042-BO) of the University of Duisburg-Essen, Germany, was obtained. 


\section{Competing interests}

The authors declare that they have no known competing financial interests or personal relationships that could have appeared to influence the work reported in this paper.

\section{Author details}

'Department of Orthopaedics, Trauma and Reconstructive Surgery, St. Marien-Hospital Mülheim a.d. Ruhr, 45468 Mülheim an der Ruhr, Germany. ${ }^{2}$ Orthopaedics and Trauma Surgery, University of Duisburg - Essen, 47057 Duisburg, Germany. ${ }^{3}$ Department of Orthopaedics, University of Saarland, 66123 Saarbrücken, Germany. ${ }^{4}$ Department of Orthopaedics, St.

Vinzenz-Krankenhaus, Schloßstraße 85, 40477 Düsseldorf, Germany.

Received: 18 March 2020 Accepted: 20 May 2020

Published online: 27 May 2020

\section{References}

1. Otto-Lambertz C, Yagdiran A, Wallscheid F, Eysel P, Jung N. Periprosthetic infection in joint replacement. Dtsch Arztebl Int. 2017;114(20):347-53. https://doi.org/10.3238/arztebl.2017.0347.

2. Parvizi J, Tan TL, Goswami K, Higuera C, Della Valle C, Chen AF, et al. The 2018 definition of periprosthetic hip and knee infection: an evidence-based and validated criteria. J Arthroplast. 2018;33(5):1309-14e1302. https://doi. org/10.1016/j.arth.2018.02.078.

3. Glehr M, Friesenbichler J, Hofmann G, Bernhardt GA, Zacherl M, Avian A, et al. Novel biomarkers to detect infection in revision hip and knee arthroplasties. Clin Orthop Relat Res. 2013;471(8):2621-8. https://doi.org/10. 1007/s11999-013-2998-3.

4. Yuan $\mathrm{K}$, Chen HL, Cui ZM. Diagnostic accuracy of C-reactive protein for periprosthetic joint infection: a meta-analysis. Surg Infect. 2014;15(5):548-59. https://doi.org/10.1089/sur.2013.066.

5. Marazzi MG, Randelli F, Brioschi M, Drago L, Romano CL, Banfi G, et al. Presepsin: a potential biomarker of PJI? A comparative analysis with known and new infection biomarkers. Int J Immunopathol Pharmacol. 2018;31: 394632017749356. https://doi.org/10.1177/0394632017749356.

6. Yuan J, Yan Y, Zhang J, Wang B, Feng J. Diagnostic accuracy of alphadefensin in periprosthetic joint infection: a systematic review and metaanalysis. Int Orthop. 2017;41(12):2447-55. https://doi.org/10.1007/s00264017-3647-3.

7. Sigmund IK, Yermak K, Perka C, Trampuz A, Renz N. Is the enzyme-linked immunosorbent assay more accurate than the lateral flow alpha defensin test for diagnosing periprosthetic joint infection? Clin Orthop Relat Res. 2018;476(8):1645-54. https://doi.org/10.1097/CORR.0000000000000336.

8. Renz N, Yermak K, Perka C, Trampuz A. Alpha defensin lateral flow test for diagnosis of periprosthetic joint infection: not a screening but a confirmatory test. J Bone Joint Surg Am. 2018;100(9):742-50. https://doi.org/ 10.2106/JBJS.17.01005.

9. Patel R, Alijanipour P, Parvizi J. Advancements in diagnosing periprosthetic joint infections after total hip and knee arthroplasty. Open Orthop J. 2016; 10:654-61. https://doi.org/10.2174/1874325001610010654

10. Ryden C, Tung HS, Nikolaev V, Engstrom A, Oldberg A. Staphylococcus aureus causing osteomyelitis binds to a nonapeptide sequence in bone sialoprotein. Biochem J. 1997;327(Pt 3):825-9. https://doi.org/10.1042/ bj3270825.

11. Tung H, Guss B, Hellman U, Persson L, Rubin K, Ryden C. A bone sialoprotein-binding protein from Staphylococcus aureus: a member of the staphylococcal Sdr family. Biochem J. 2000;345(Pt 3):611-9.

12. Ryden C, Maxe I, Franzen A, Ljungh A, Heinegard D, Rubin K. Selective binding of bone matrix sialoprotein to Staphylococcus aureus in osteomyelitis. Lancet. 1987;2(8557):515. https://doi.org/10.1016/s01406736(87)91830-7.

13. Persson L, Johansson C, Ryden C. Antibodies to Staphylococcus aureus bone sialoprotein-binding protein indicate infectious osteomyelitis. Clin Vaccine Immunol. 2009;16(6):949-52. https://doi.org/10.1128/CVI.00442-08.

14. Bilgen $\mathrm{O}$, Atici T, Durak K, Karaeminogullari BMS. C-reactive protein values and erythrocyte sedimentation rates after total hip and total knee arthroplasty. J Int Med Res. 2001;29(1):7-12. https://doi.org/10.1177/ 147323000102900102.

15. Larsson S, Thelander U, Friberg S. C-reactive protein (CRP) levels after elective orthopedic surgery. Clin Orthop Relat Res. 1992;275:237-42.
16. Bingham JS, Salib CG, McQuivey K, Temkit M, Spangehl MJ. An evidencebased clinical prediction algorithm for the musculoskeletal infection society minor criteria. J Arthroplast. 2018;33(9):2993-6. https://doi.org/10.1016/j.arth. 2018.04.047.

17. Parvizi J, Zmistowski B, Berbari EF, Bauer TW, Springer BD, Della Valle CJ, et al. New definition for periprosthetic joint infection: from the workgroup of the musculoskeletal infection society. Clin Orthop Relat Res. 2011;469(11): 2992-4. https://doi.org/10.1007/s11999-011-2102-9.

18. Shohat N, Tan TL, Della Valle CJ, Calkins TE, George J, Higuera C, et al. Development and validation of an evidence-based algorithm for diagnosing periprosthetic joint infection. J Arthroplast. 2019;34(11):2730-6e2731. https:// doi.org/10.1016/j.arth.2019.06.016.

19. Wang C, Wang Q, Li R, Qin J, Song L, Zhang Q, et al. LTF, PRTN3, and MNDA in synovial fluid as promising biomarkers for periprosthetic joint infection: identification by quadrupole orbital-trap mass spectrometry. J Bone Joint Surg Am. 2019;101(24):2226-34. https://doi.org/10.2106/JBJS.18.01483.

20. Vergara A, Fernandez-Pittol MJ, Munoz-Mahamud E, Morata L, Bosch J, Vila J, et al. Evaluation of lipocalin-2 as a biomarker of periprosthetic joint infection. J Arthroplast. 2019;34(1):123-5. https://doi.org/10.1016/j.arth.2018. 09.047.

21. Plate A, Anagnostopoulos A, Glanzmann J, Stadler L, Weigelt L, Sutter R, et al. Synovial C-reactive protein features high negative predictive value but is not useful as a single diagnostic parameter in suspected periprosthetic joint infection (PJI). J Inf Secur. 2019;78(6):439-44. https://doi.org/10.1016/j. jinf.2019.04.003.

22. Fisher LW, McBride OW, Termine JD, Young MF. Human bone sialoprotein Deduced protein sequence and chromosomal localization. J Biol Chem. 1990;265(4):2347-51.

23. Ryden C, Yacoub A, Hirsch G, Wendel M, Oldberg A, Ljungh A. Binding of bone sialoprotein to Staphylococcus epidermidis isolated from a patient with chronic recurrent multifocal osteomyelitis. J Infect Dis. 1990;161(4):8145. https://doi.org/10.1093/infdis/161.4.814.

24. Campoccia D, Speziale P, Ravaioli S, Cangini I, Rindi S, Pirini V, et al. The presence of both bone sialoprotein-binding protein gene and collagen adhesin gene as a typical virulence trait of the major epidemic cluster in isolates from orthopedic implant infections. Biomaterials. 2009;30(34):66218. https://doi.org/10.1016/j.biomaterials.2009.08.032.

25. Wang C, Wang Q, Li R, Duan JY, Wang CB. Synovial fluid C-reactive protein as a diagnostic marker for periprosthetic joint infection: a systematic review and meta-analysis. Chin Med J. 2016;129(16):1987-93. https://doi.org/10. 4103/0366-6999.187857.

26. Chen Y, Kang X, Tao J, Zhang Y, Ying C, Lin W. Reliability of synovial fluid alpha-defensin and leukocyte esterase in diagnosing periprosthetic joint infection (PJI): a systematic review and meta-analysis. J Orthop Surg Res. 2019;14(1):453. https://doi.org/10.1186/s13018-019-1395-3.

27. Tahta M, Simsek ME, Isik C, Akkaya M, Gursoy S, Bozkurt M. Does inflammatory joint diseases affect the accuracy of infection biomarkers in patients with periprosthetic joint infections? A prospective comparative reliability study. J Orthop Sci. 2019;24(2):286-9. https://doi.org/10.1016/j.jos. 2018.08.022.

28. Saleh A, George J, Faour M, Klika AK, Higuera CA. Serum biomarkers in periprosthetic joint infections. Bone Joint Res. 2018;7(1):85-93. https://doi. org/10.1302/2046-3758.71.BJR-2017-0323.

29. Harrison M. Erythrocyte sedimentation rate and C-reactive protein. Aust Prescr. 2015;38(3):93-4. https://doi.org/10.18773/austprescr.2015.034.

30. Ridker PM. C-reactive protein: eighty years from discovery to emergence as a major risk marker for cardiovascular disease. Clin Chem. 2009;55(2):209-15. https://doi.org/10.1373/clinchem.2008.119214.

\section{Publisher's Note}

Springer Nature remains neutral with regard to jurisdictional claims in published maps and institutional affiliations. 